\title{
A novel approach to tackling the 'benzodiazepine problem' in general practice
}

\author{
MAUREen O'LEARY, Senior Registrar, Whiteley Wood Clinic, Sheffield
}

With growing concern - both professional and lay (Ashton, 1986; Lacey \& Woodward, 1985) - about the adverse effects of long-term benzodiazepine use, many GPs are wondering how best to tackle the problem. In this paper, I describe a strategy adopted by a GP, CPN and psychiatrist (the author). This strategy centred around a meeting of all patients who had been taking benzodiazepines for over six months.

\section{Background to the meeting}

Since 1984 any patient who was prescribed benzodiazepines (for any purpose including night sedation) for over six months was entered on a 'disease register' of the practice. This was done to organise more effective education and follow-up of patients, and assist a research project in which chronic benzodiazepine users are compared to a matched population.

The current GPs at the practice had 'inherited' a large number of chronic benzodiazepine users many of whom are in their sixties and seventies. Throughout the 1980s the two GPs used individual consultations to educate users about benzodiazepines and to encourage withdrawal. Despite this approach many patients continued to use these drugs.

In 1988 GPs were inundated with recommendations about how to deal with chronic benzodiazepine users (Rodrigo et al, 1988). This gave urgency to a reappraisal of the management of this group of patients. A decision was taken to supplement the 'individual' approach with a 'group' approach.

A meeting was organised with the aims of increasing the level of knowledge of chronic users, and generating an opportunity for chronic users to meet and share their experiences of these drugs. The possible outcomes of this meeting were hypothetical and, therefore, exciting. It was hoped this excitement would spur us on to greater efforts to tackle 'the problem'.

\section{Organisation of the meeting}

The 'disease register' was updated. This, in itself, was a useful experience. Of the 186 patients entered on the register since 1984 , only 92 were currently using benzodiazepines. Unfortunately, this attrition was not all due to patients stopping benzodiazepines. Fourteen users had died, 32 had moved to another GP, but 48 users had withdrawn from benzodiazepines. It was heartening that $25.7 \%$ of chronic users had successfully withdrawn from benzodiazepines particularly as the population is elderly and had been taking the drugs for many years. Of the 92 current users, six were not invited to the meeting, on the advice of the GPs (three for physical health reasons and three on mental health grounds).

The remaining 86 patients were sent a personal letter from both GPs inviting them to the meeting. In addition, they were sent a copy of the MIND leaflet Minor Tranquillisers: Hard Facts Hard Choices which they were asked to read prior to the meeting. The meeting was arranged to maximise attendance. It was held at a familiar place - the surgery - at $7 \mathrm{pm}$ in May - in daylight hours. Transport was offered if necessary - but none was requested.

\section{The meeting}

It was held in a large waiting room at the surgery. Of the 86 people invited, 32 people attended (37\%). In addition, three spouses accompanied their partners, one spouse came on behalf of her partner and one daughter of a user came. In all 37 people turned up. Those attending were asked to register their names, state whether they wanted to come off benzodiazepines, and state whether they wanted to discuss the matter more fully with the GP.

At the beginning of the meeting, one of the GPs gave a short speech. He introduced the CPN and psychiatrist and drew attention to the leaflet which had been circulated. Most people had read it and found it informative and easy to understand. The speech supplied a brief historical perspective on the introduction of the benzodiazepines, the explosions of their use in the 1960s and early 1970s and the gradual recognition by doctors and users of their long-term disadvantages. The GP used his speech to state firmly that he and his partner wanted as many patients as possible to come off benzodiazepines. However, they were not going to refuse to prescribe these tablets for patients who were not ready to make that choice. They wanted each patient to have enough information to make an informed decision. The GP then gave general guidelines about reduction 
regimes and possible withdrawal symptoms. He stressed he and his partner's commitment to supporting patients through withdrawal.

After a short and noisy tea-break, the meeting was open for informal discussion.

Many fears were expressed about withdrawal. The most common fear was of sleepless nights. Several users said their sleep was poor despite benzodiazepines and feared it might deteriorate further. Other users described their efforts at reduction-some of which were quite successful-and gave hope to others. A few indicated strongly that the tablets were useful to them and they intended to continue using them. Other users asked factual questions about the psychopharmacology of the drugs.

At the end of the meeting an announcement was made, asking anyone interested in participating in a support group to stay behind; six ( $18 \%$ of those attending who were on tranquillisers) did so. A time and venue for a meeting with the CPN was arranged; three of the six were already members of a self-help agorophobic group which has been running at the practice for several years.

Those users who had indicated an interest in reducing or coming off benzodiazepines were informed of the first meeting of this potential support group.

Of the 32 users attending the meeting, $13(40 \%)$ did not want to come off benzodiazepines, $12(37.5 \%)$ did, two $(6 \%)$ wanted to reduce their intake and two (6\%) wanted more time to discuss it with their GP, and three $(9 \%)$ did not comment.

Of the 16 users informed about the follow-up meeting with the CPN, 15 attended $(93 \%)$. There were another two meetings. The final meeting was attended by the four members of the agorophobic group and the CPN. At this stage, they decided to continue meeting in the agorophobic group as they had done for years.

\section{Comment}

The two aims of this project were to increase the level of knowledge of chronic users, and to generate an opportunity for chronic users to meet and share their experiences. It is likely that the first aim was achieved. Even patients who chose not to attend the meeting had received the MIND leaflet - which they may have read. Those patients who came to the meeting are likely to have a clearer idea of side effects of chronic benzodizepine use, how their GPs view the problem and sensible ways of coming off tranquillisers. The second aim was certainly achieved for those who chose to attend (37\%). The meeting was lively and people were actively listening to each other as well as contributing to the discussion. Whether achievement of these two aims helps to reduce the number of chronic users will be difficult to judge. Many factors are likely to influence this, apart from the meeting-in particular the increasing media discussions (BBC TV, 1988) of the problems of chronic benzodiazepine use.

It is interesting that the potential support group withered on the vine. This is particularly suprising as $93 \%$ of users who expressed an interest in withdrawal, came to the first follow-up meeting. Users were informed about this meeting by a letter from the GP. This may have been important in producing such a high turn out. The three follow-up meetings with the CPN were unstructured. Structured meetings may have allowed people to attend until sufficient trust had been built up for a less structured group. The CPN was unknown to most of the users prior to the first meeting. He, therefore, did not command the loyalty that the GP or other workers in the practice may have. It is possible that the powerful subgroup of four agorophobics who had met for several years, made it more difficult for a new group to gel. This is speculation, but if we repeat the exercise, more thought will go into the form and content of group sessions. Perhaps users need to be consulted about which professional should facilitate the support group.

\section{Plans for the future}

A further meeting is planned for May 1989. Invitations will be issued to all chronic users and those who have managed to withdraw over the year. Hopefully ex-users will choose to attend in order to educate, encourage and support those still on benzodiazepines. A viable support group of users wishing to withdraw and ex-users may emerge.

\section{References}

Ashton, H. (1986) Adverse effects of prolonged benzodiazepine use. Adverse Drug Reaction Bulletin, 118, 440-443.

BBC TV (1988) Tranquilliser initiative. Day Time Live. BBC, Pebble Mill, Birmingham. November 1988.

LACEY, R. \& WOODWARD, S. (1985) That's Life! Survey on Tranquillisers (with a foreword by Esther Rantzen). London: BBC in association with MIND.

MIND (1987) Special Report: Minor Tranquillisers: Hard Facts Hard Choices. London: MIND Publications.

Rodrigo, E. K., King, M. B. \& Williams, P. (1988) Health of long-term benzodiazepine users. British Medical Journal, 296, 603-605. 